\title{
Ionization Depth Distribution in EPMA: Improvement of the Random Walk Model
}

\author{
G. Castellano, ${ }^{*}$ S. Segui and J. Trincavelli \\ FaMAF, Universidad Nacional de Córdoba, Córdoba, Argentina, and Consejo Nacional de Investigaciones Científicas y \\ Técnicas de la República Argentina
}

\begin{abstract}
A model for the parameter $\gamma$ involved in Packwood and Brown's expression for the ionization depth distribution $\phi(\rho z)$ in EPMA is developed. Assuming that the electrons perform a random walk within the sample, the parameter $\gamma$ is related to the probability of finding an electron in the surface layer after a large number of steps. Despite the simplicity of the model, the resulting $\gamma$ values produce a very good description of $\phi(\rho z)$ distributions obtained through Monte Carlo simulations and experimental data. (C) 1998 John Wiley \& Sons, Ltd.
\end{abstract}

X-Ray Spectrom. 27, 293-298 (1998)

\section{INTRODUCTION}

When quantifying a sample of mass density $\rho$ in electron probe microanalysis (EPMA), the knowledge of the depth distribution of primary ionizations $\phi(\rho z)$ is very useful, since characteristic x-ray emission is mainly governed by them. A large number of models have been developed to describe $\phi(\rho z)$, on the basis of physical principles or by means of empirical or semi-empirical procedures. Among the first ones, the Gaussian model by Packwood and Brown ${ }^{1}$ was developed after a careful analysis of experimental $\phi(\rho z)$ curves. They observed a Gaussian behavior starting at a certain depth very close to the surface, suggesting that some random process governs the interaction of electrons within the sample. Associating this randomness with elastic scattering, they proposed a model in which electrons perform a random walk. The resulting normal distribution must be modified close to the surface by means of a transient function which takes into account the initially forward-privileged direction of electrons entering the sample. Packwood and Brown proposed an exponential as a transient function, arriving at the following expression for $\phi(\rho z)$ :

$$
\begin{aligned}
\phi(\rho z)= & \exp \left[-\alpha^{2}(\rho z)^{2}\right] \\
& \times\left\{\gamma-\left(\gamma-\phi_{0}\right) \exp [-\beta \rho z]\right\}
\end{aligned}
$$

A number of models have been developed in order to describe parameters $\alpha, \beta, \gamma$ and $\phi_{0}$ as functions of the incident electron energy $E_{0}$, the elements in the sample and the critical energy $E_{\mathrm{c}}$ of the shell of interest. In the

\footnotetext{
* Correspondence to: G. Castellano, FaMAF, Universidad Nacional de Córdoba, Ciudad Universitaría 5000 Córdoba, Argentina.

Contract/grant sponsor: Consejo Nacional de Investigaciones Científicas y Técnicas de la Républica Argentina.

Contract/grant sponsor: Consejo de Investigaciones Científicas y Technológicas de la Provincia de Córdoba.

Contract/grant sponsor: Secretaría de Ciencia y Técnica de la Universidad Nacional de Córdoba.
}

original work, Packwood and Brown ${ }^{1}$ related the parameter $\alpha$ to the stopping power and the scattering properties of the irradiated material, achieving an adequate description. However, the remaining parameters were not properly studied, giving rise to successive analyses.

Bastin and co-workers ${ }^{2-4}$ optimized these parameters for certain sets of binary samples, giving no physical support for the expressions that they obtained. Riveros and co-workers ${ }^{5-7}$ tried to develop expressions sustained on basical principles, intending to describe adequately a wide range of experimental situations. On the other hand, the surface ionization $\phi_{0}$ has been repeatedly studied, ${ }^{8-10}$ reproducing the experimental data each time more accurately.

In this work, a model for the parameter $\gamma$ is presented, intended to overcome the inappropriate descriptions given by the existing expressions. ${ }^{4,5}$ The starting point is the assumption that electrons in an infinite target perform an isotropical random walk, $\gamma$ being the amplitude of the ionization depth distribution corresponding to that situation. The model is used to describe the $\phi(\rho z)$ curves obtained by Monte Carlo simulation and experimental data for several elements and incident voltages. A comparison with other expressions is also given.

\section{DESCRIPTION OF THE MODEL}

According to Packwood and Brown, ${ }^{1}$ the probability of finding an electron in a layer of mass thickness $\Delta \rho z$ at depth $\rho z$ after a large number $M$ of isotropic steps of length $\rho \lambda$ is

$$
P(\rho z) \Delta \rho z=\frac{1}{\sqrt{2 \pi M} \rho \lambda} \exp \left[-\frac{(\rho z)^{2}}{2 M(\rho \lambda)^{2}}\right] \Delta \rho z
$$

where $\rho \lambda$ is associated with the mean free path of the electrons in the sample. These assumptions imply a 

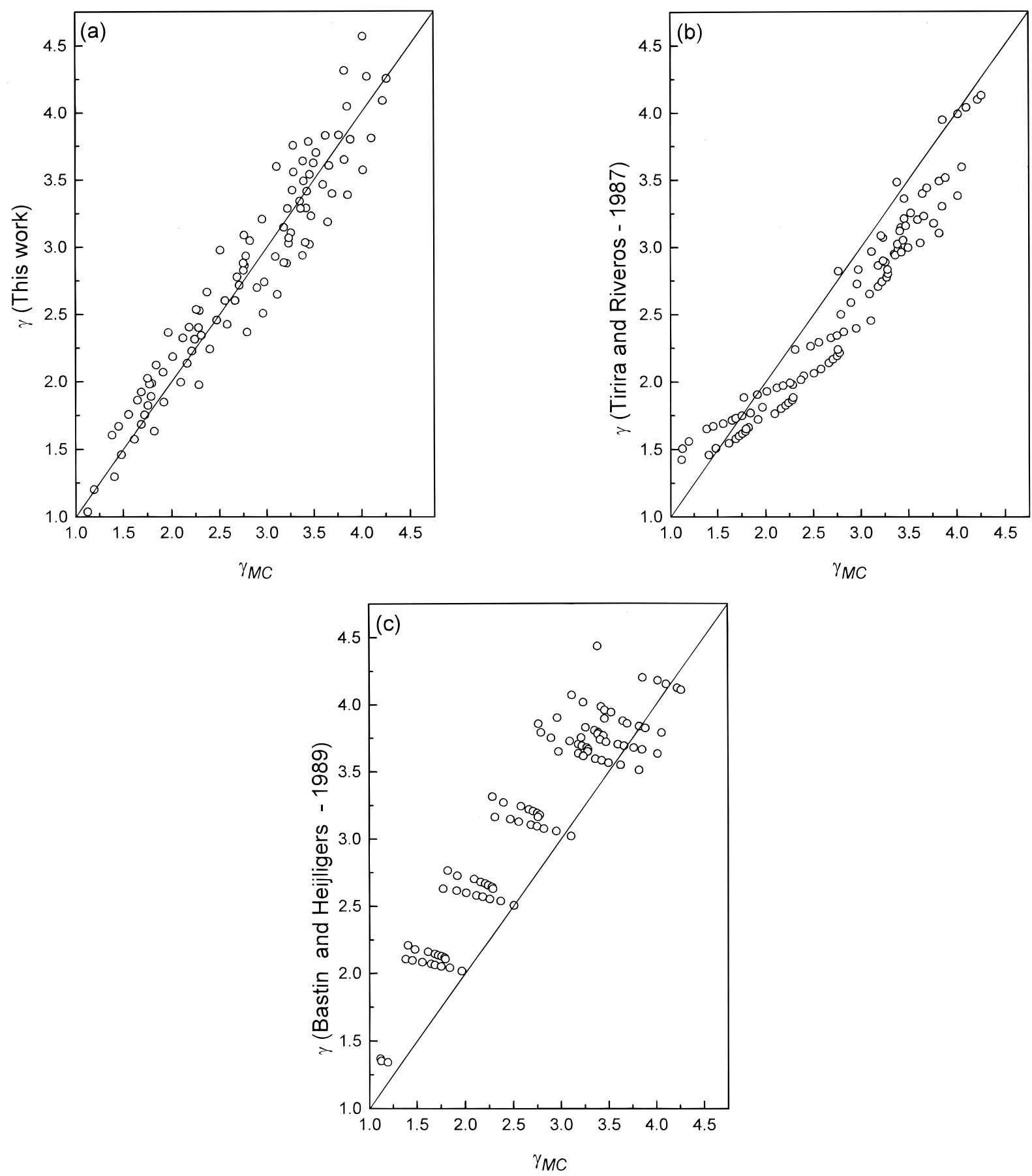

Figure 1. Comparison of $\gamma$ values obtained with the models given (a) in this work, (b) by Tirira and Riveros and (c) by Bastin and Heijligers with those corresponding to Monte Carlo simulation for the depth distribution $\phi(\rho z)$.

Gaussian ionization depth distribution with the same width of the distribution $P(\rho z)$. Comparison with Eqn (1) leads to

$$
\alpha=\frac{1}{\sqrt{2 M} \rho \lambda}
$$

The number of steps $M$ is obtained by dividing the useful energy interval of electrons $\left(E_{0}-E_{\mathrm{c}}\right)$ by the average energy loss $\Delta E$ in each step of length $\rho \lambda$ :

$$
M=\frac{E_{0}-E_{\mathrm{c}}}{S \rho \lambda}
$$

In this expression $\Delta E$ has been replaced by $S \rho \lambda$, where $S$ is the stopping power, following a continuous slowing down approximation.

The knowledge of the amplitude of the Gaussian depth distribution of electrons $P(\rho z)$ allows the assessment of $\gamma$, which is the amplitude of the ionization depth distribution in an infinite sample where all electron path directions have the same probability. The amplitude of $P(\rho z)$, i.e. the probability density of finding an electron at $\rho z=0$ after $M$ steps, is $\alpha / \sqrt{\pi}$. With the purpose of obtaining the probability $P^{\prime} \Delta \rho z$ for that electron to ionize the element of interest in the $M$ th step, it is necessary to multiply by the corresponding cross- 

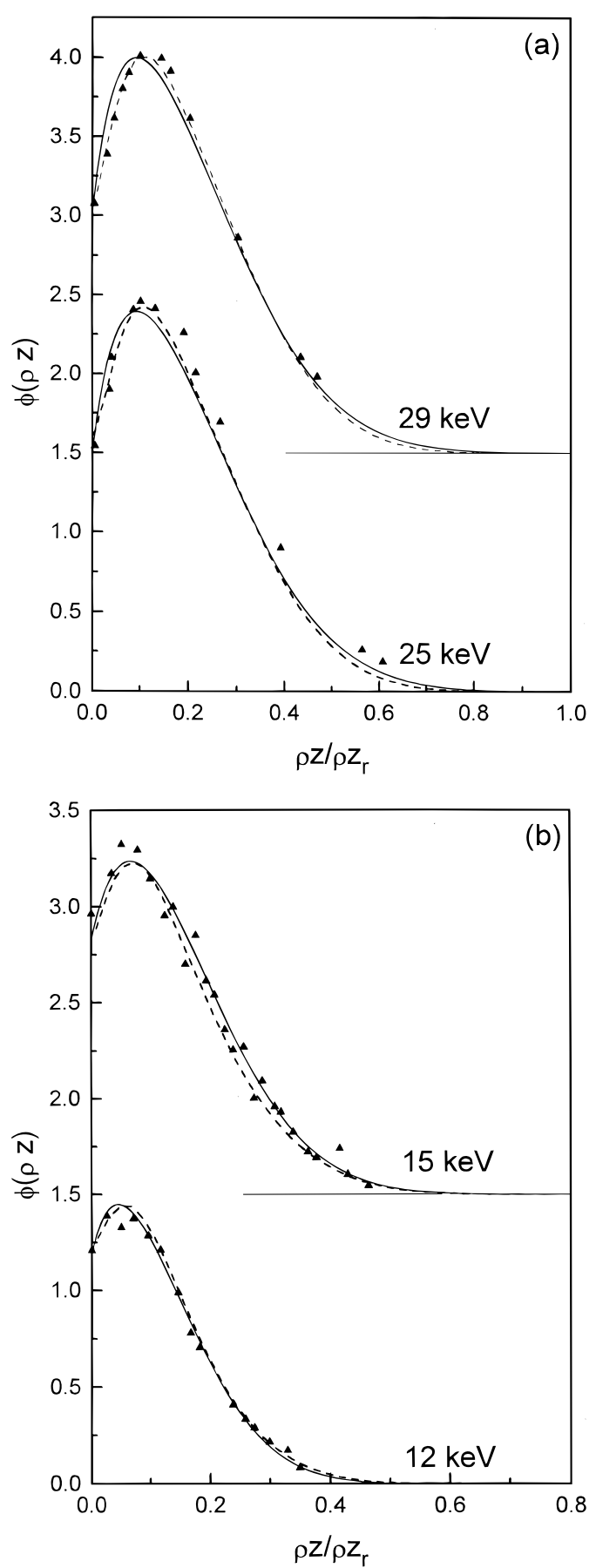

Figure 2. K ionization depth distributions $\phi(\rho z)$ for (a) titanium and (b) nickel. Triangles, experimental data;26,27 dashed line, Monte Carlo simulations; full line, Gaussian model with parameters $\phi_{0}$ by Segui et al., ${ }^{10} \alpha$ by Packwood and Brown, ${ }^{1} \gamma$ given in Eqn (6) and $\beta$ fitted to simulations. On the abscissas the mass depth $\rho z$ has been normalized with the mass range $\rho z_{r}$ using the expression for the stopping power given in Ref. 28.

section $Q$, the pathlength $\lambda$ traversed in that step and the number of atoms per unit volume, $N_{0} \rho / A$, where $N_{0}$ is Avogadro's number and $A$ the atomic weight. Since $\gamma$ accounts for the probability of ionizing along the $M$ steps performed - not just the last one- a convolution should be assessed, considering all the possible steps between 1 and $M$, in addition to the probability of ionizing in each one of them. In the search for simplicity, the probability of ionizing in the $M$ th step will be multiplied by $M$, assuming that all steps have an ionizing efficiency equal to the last one. This leads to

$$
P^{\prime} \Delta \rho z=\frac{\alpha}{\sqrt{\pi}} \frac{N_{0}}{A} Q \rho \lambda M \Delta \rho z
$$

So far, no attention has been paid to the dependence of the ionization cross-section and the mean free path on the electron energy. In order to maintain the random walk formalism introduced by Packwood and Brown, all steps must be considered of the same length; this is equivalent to evaluating $\rho \lambda$ at some energy $\widetilde{E}$, which will also be used to compute the cross-section.

On the other hand, $\phi(\rho z)$ is defined as the number of ionizations produced between $\rho z$ and $\rho z+\Delta \rho z$ within a semi-infinite sample, divided by the number of ionizations occurring in an isolated thin layer of width $\Delta \rho z$, irradiated under the same experimental conditions. Bearing this definition in mind, the amplitude $\gamma$ of the ionization depth distribution corresponding to the random walk is obtained by dividing the previous expression by the number of ionizations produced in the isolated layer, $\left(N_{0} / A\right) Q\left(E_{0}\right) \Delta \rho z$. This leads to

$$
\gamma=\sqrt{\frac{M}{2 \pi}} \frac{Q(\tilde{E})}{Q\left(E_{0}\right)}
$$

In this work, following the assumptions made by Packwood and Brown in their original paper, ${ }^{1}$ the value chosen for $\widetilde{E}$ is $E_{0}$, thus arriving at the following simplified expression:

$$
\gamma=\sqrt{\frac{M}{2 \pi}}
$$

In order to calculate $M$, the expression given by Bethe and Ashkin ${ }^{11}$ was chosen for $S$, including a shell effect correction for the mean ionization potential $J{ }^{12}$ The mean free path $\rho \lambda$ was evaluated from Rutherford's cross-section, with screening corrections according to Bishop. ${ }^{13}$ The dependence of $\gamma$ on $E_{0}, E_{\mathrm{c}}$ and the atomic number $Z$ results:

$$
\gamma=1.8227 \sqrt{\frac{\left(E_{0}-E_{\mathrm{c}}\right) Z^{0.33}}{E_{0}^{0.5} \ln \left(1.166 E_{0} / J\right)}}
$$

In this expression, the correction proposed by Packwood and Brown ${ }^{1}$ for the $E_{0}$ dependence of the screening factor has been included.

The constant factor of Eqn (6) is a parameter which had to be optimized because of the simplifications considered. To this end, a large number of Monte Carlo simulations have been performed in order to obtain $\phi(\rho z)$ values using the PENELOPE routine package. ${ }^{14,15}$ This package keeps track of both the position and energy of electrons within the sample, while the ionizations produced are registered. The probability of each interaction occuring is randomly generated by means of highly reliable cross-sections. ${ }^{16-18}$ This simulation method has been widely checked ${ }^{19-22}$ and, in particular, it has been shown to describe $\phi(\rho z)$ adequately. ${ }^{23}$ Gaussian distributions [Eqn (1)] were fitted to the data thus generated, with the parameters $\alpha$ given by Packwood and Brown ${ }^{1}$ and $\phi_{0}$ by Segui et al., ${ }^{10}$ thus producing a number of values $\gamma_{\mathrm{MC}}$ and $\beta_{\mathrm{MC}}$. Finally, the constant of Eqn (6) was obtained by plot- 

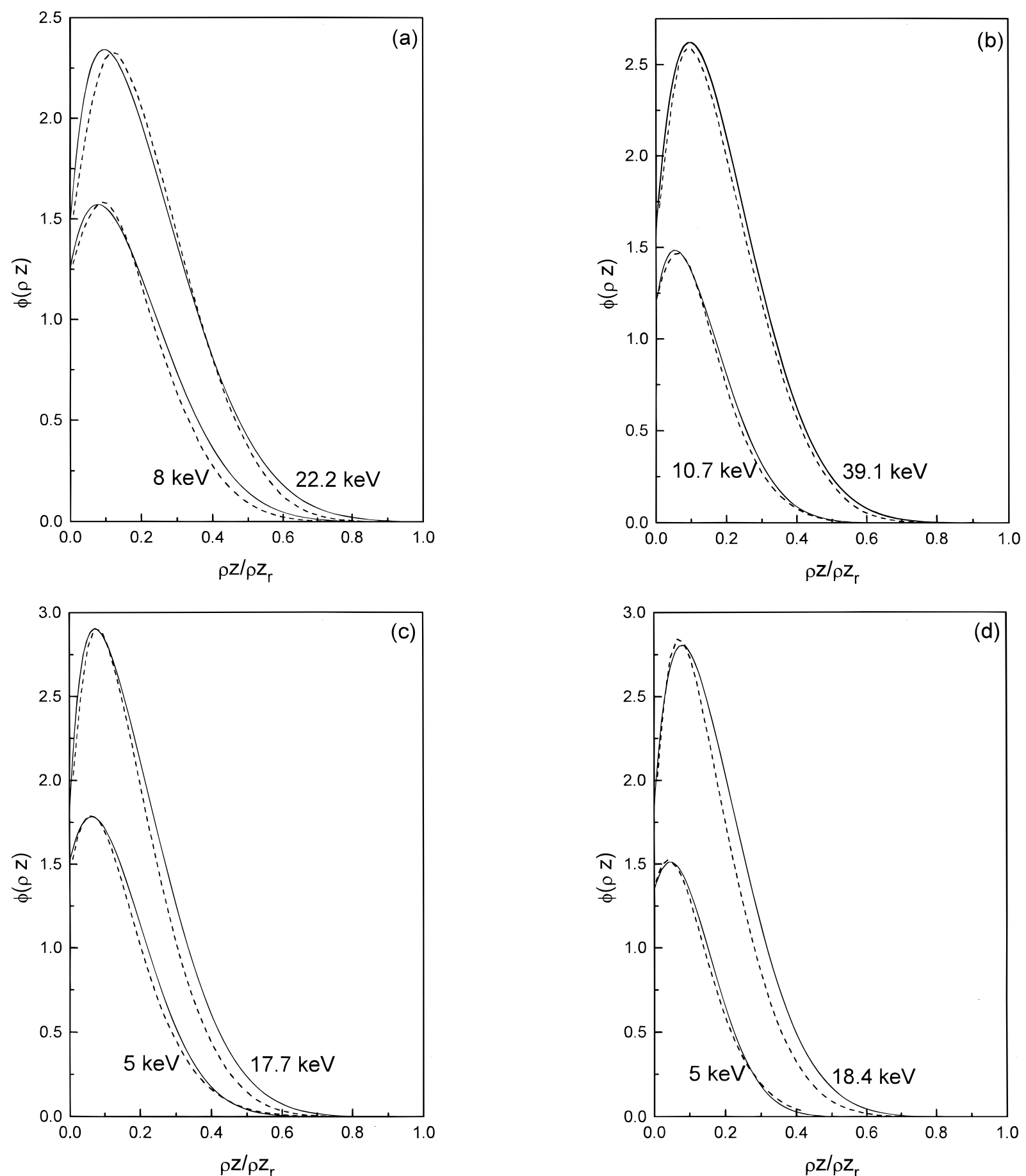

Figure 3. Ionization depth distributions $\phi(\rho z)$ for (a) calcium and (b) iron K edges and (c) molybdenum and (d) silver L edges. Dashed line, Monte Carlo simulations; full line, Gaussian model with parameters $\phi_{0}$ by Segui et al., ${ }^{10} \alpha$ by Packwood and Brown, ${ }^{1} \gamma$ given in Eqn (6) and $\beta$ fitted to simulations. On the abscissas the mass depth $\rho z$ has been normalized with the mass range $\rho z_{\mathrm{r}}$ using the expression for the stopping power given in Ref. 28.

ting the developed expression vs. $\gamma_{\mathrm{MC}}$ and fitting a straight line.

It must be noted that this constant differs from unity since the physical phenomena governing this diffusion process have been simplified in order to reduce the calculations. In particular, the approximation $\widetilde{E}=E_{0}$ may mainly influence those cases for which $E_{0}$ is very close to $E_{\mathrm{c}}$, but the random walk assumptions lose validity precisely in these cases, since the number of steps is far from large.

\section{RESULTS AND COMPARISON WITH OTHER MODELS}

The Monte Carlo simulations for $\phi(\rho z)$ distributions were obtained for atomic numbers ranging from 16 to 35 for $\mathrm{K}$ edges, and from 35 to 73 for $\mathrm{L}_{\mathrm{III}}$ edges; incident overvoltages ranged from 1.5 to 15 , provided that the energies involved were reasonable EPMA voltages (up to $50 \mathrm{keV}$ ). Figure 1(a) shows values for $\gamma$ obtained with 
the model developed here vs. $\gamma_{\mathrm{MC}}$ values. A linear dependence can be observed, with a zero intercept on the ordinate.

Packwood and Brown ${ }^{1}$ tried to give an expression for $\gamma$ on the basis of a random-walk model. However, they failed in using parameters (such as the backscattering coefficient $\eta$ ) corresponding to the real situation for a collimated electron beam, and they also evaluated inadequately the mean electron path in the surface layer. Corrections were then suggested by Tirira and Riveros, ${ }^{5}$ who chose a better approximation for the mean path and for the mean ionization potential. The following expression was obtained:

$$
\gamma=(1+\eta) U_{0} \frac{\ln U_{0}}{U_{0}-1}
$$

where $U_{0}=E_{0} / E_{\mathrm{c}}$, and for the ionization cross-section the Bethe expression ${ }^{24}$ was used including the modifications made by Green and Cosslett. ${ }^{25}$ However, they still continued to use the parameter $\eta$ as though the situation took into account non-isotropic initial directions for the electrons - which is not the case under analysis. In Fig. 1(b) a comparison between $\gamma$ values obtained with this model and simulation values is shown.

In a completely different fashion, Bastin and Heijligers ${ }^{4}$ carried out a series of fits and mathematical optimizations to the parameters of the $\phi(\rho z)$ distribution; specific values for the generated intensity were then obtained and contrasted with a set of microanalysis data for standard samples. Their resulting expression is

$$
\gamma= \begin{cases}3.98352 U_{0}^{-0.0516861} & \text { if } U_{0} \leqslant 6 \\ \times\left(1.276233-U_{0}^{\left.-1.25558 Z^{-0.1424549}\right)}\right. & \\ 2.814333 U_{0}^{0.262702 Z^{-0.1614454}} & \text { if } U_{0}>6\end{cases}
$$

This function should be multiplied by $E_{\mathrm{c}} /(-0.041878$ $\left.+1.05975 E_{\mathrm{c}}\right)$ when $Z \leq 9$. Values obtained by means of this expression are plotted in Fig. 1(c).

As can be observed in Fig. 1, the expressions by Tirira and Riveros ${ }^{5}$ and by Bastin and Heijligers ${ }^{4}$ show dependences on some of the parameters $\left(E_{0}, E_{\mathrm{c}}\right.$ or $\left.Z\right)$, whereas the equation suggested in this work does not exhibit any systematic deviation from the straight line $45^{\circ}$ slope. In particular, the complicated function proposed by Bastin and Heijligers shows a strong dependence on the atomic number.

In Fig. 2, $\phi(\rho z)$ distributions obtained from Monte Carlo simulations and measurements ${ }^{26,27}$ are shown for titanium and nickel $\mathrm{K}$ edges. The mass depth $\rho z$ has been normalized with the mass range $\rho z_{\mathrm{r}}$ using the expression for the stopping power given in Ref. 28. Gaussian curves are also plotted using parameters $\phi_{0}$ given by Segui et al., ${ }^{10} \alpha$ by Packwood and Brown ${ }^{1}$ and $\gamma$ developed in the present work, whilst the parameter $\beta$ was computed to fit the simulated $\phi(\rho z)$ data close to the surface. It can be seen that experimental data are adequately predicted by means of Monte Carlo simulations, and also by the expression involving the parameter $\gamma$ developed here. Although this looks like good agreement, it should be noted that reliable experimental data for $\phi(\rho z)$ are not always available. Absorption corrections used for obtaining values generated within the sample are hardly ever provided along with experimental data. In particular, different models for mass absorption coefficients may produce very different values, which may result in large differences for $\phi(\rho z)$ values at large depths. On the other hand, additional uncertainties may arise when building up samples for $\phi(\rho z)$ measurements. Fortunately, simulations produced with the PENELOPE package have proved to describe properly not only the $\phi(\rho z)$ function, but even complete spectra measured in EPMA, ${ }^{20}$ accounting for the overall physical processes. For these reasons, Monte Carlo simulations were used to test the expression for $\phi(\rho z)$ with the model for $\gamma$ proposed here. These comparisons are shown in Fig. 3(a) and (b), where calcium and iron $\phi(\rho z)$ curves are plotted for $\mathrm{K}$ ionizations. Similarly, in Fig. 3(c) and (d) $\mathrm{L}_{\text {III }}$ edge depth distributions for molybdenum and silver are displayed.

In all cases, the ionization cross-sections given by Mayol and Salvat ${ }^{29}$ were used for studying $\mathrm{K}$ ionizations. For $\mathrm{L}$ ionizations, cross-sections suggested by Pouchou and Pichoir ${ }^{30}$ were implemented.

As shown in the plots, for different overvoltages the predictions of Eqn (6) are satisfactory, especially for K edges. Whereas Mayol and Salvat developed their model on the basis of careful physical considerations, the small deviations observed for $L$ edges might arise from the use of a more simplified cross-section model. Anyway, these deviations generally correspond to regions away from the surface, so these differences will not influence quantifications.

\section{CONCLUSION}

The model for the parameter $\gamma$ proposed in this work is the only one developed entirely from considerations based on a random walk of the electrons within the sample, in accordance with the Gaussian description for $\phi(\rho z)$ given by Packwood and Brown. ${ }^{1}$

The simplifications assumed produce a simple analytical function for $\gamma$; nevertheless, the expression obtained shows very good agreement with experimental data and highly reliable Monte Carlo simulation values. ${ }^{23}$ On the other hand, the model proposed in this work exhibits a better performance than previous models in the range of situations studied; this fact is particularly evidenced when making a comparison with the highly complicated expression given by Bastin and Heijligers. $^{4}$

Finally, it should be mentioned that further work must be carried out on the remaining parameters $\alpha$ and $\beta$ in order to achieve a global analytical description for $\phi(\rho z)$, on the basis of physical considerations. This would give rise to a quantification method valid for most experimental situations, not being restricted to a given set of microanalyses, as in the models which arise from mathematical optimizations. 


\section{Acknowledgements}

The authors acknowledge financial support from the Consejo Nacional de Investigaciones Científicas y Técnicas de la República Argentina, the Consejo de Investigaciones Científicas y Technológicas de la Provincia de Córdoba and the Secretaría de Ciencia y Técnica de la Universidad Nacional de Córdoba. Special thanks are due to Professor J. A. Riveros for his invaluable advice.

\section{REFERENCES}

1. R. Packwood and J. Brown, X-Ray Spectrom, 10, 138 (1981).

2. G. Bastin, F. Van Loo and H. Heijligers, X-Ray Spectrom. 13, 91 (1984)

3. G. Bastin, H. Heijlgers and F. Van Loo, Scanning 8, 45 (1986).

4. G. Bastin, H. Heijligers, Rep. Eindhoven Univ. Technol. p. 73 (1990).

5. J. Tirira and J. Riveros, X-Ray Spectrom. 16, 27 (1986).

6. J. Tirira, M. del Giorgio and J. Riveros, X-Ray Spectrom. 16, 243 (1987)

7 M. del Giorgio, J. Trincavelli and A. Riveros, X-Ray Spectrom. 19, 261 (1990)

8. C. Merlet, X-Ray Spectrom. 21, 229 (1992)

9. W. Rehbach and P. Karduck, Microbeam Analysis, p. 249. San Francisco Press, San Francisco (1988).

10. S. Segui, G. Castellano, J. Trincavelli and J. Riveros, $X$-Ray Spectrom. 25, 110 (1996).

11. H. Bethe and J. Ashkin, Exp. Nucl. Phys. 1, 252 (1953).

12. H. Livingston and H. Bethe, Rev. Mod. Phys. 9, 245 (1937).

13. H. Bishop, Br. J. Appl. Phys. 18, 703 (1967).

14. J. Baró, J. Sempau, J. Fernández-Varea and F. Salvat, Nucl. Instrum. Methods, Sect. B 100, 31 (1995).

15. J. Sempau, E. Acosta, J. Baró, J. Fernández-Varea and F. Salvat, Nucl. Instrum. Methods, Sect. B submitted for publication.

16. F. Salvat and J. Fernández-Varea, Nucl. Instrum. Methods, Sect. B 73, 447 (1993).

17. D. Liljequist, J. Phys. D. 16, 1567 (1983).

18. J. Baró, J. Sempau, J. Fernández-Varea and F. Salvat, Nucl. Instrum. Methods, Sect B 84, 465 (1994).

19. E. Acosta, E. Coleoni, G. Castellano, J. Riveros, J. Fernández and F. Salvat, Scanning Micros. 10, 625 (1996).
20. E. Acosta, X. Llovet, E. Coleoni, J. Riveros and F. Salvat Avances en Análisis por Técnicas de Rayos X, Vol. IX, Proceedings of the $V$ Seminario Latinoamericano de Análisis por Técnicas de Rayos $X$, edited by $\mathrm{G}$. Castellano, J. Riveros, $\mathrm{H}$. Sánchez, G. Stutz and J. Trincavelli, p. 138. Universidad Nacional de Córdoba, Córdoba, Argentina (1997).

21. J. Britch, E. Coleoni, E. Acosta, A. Santiago and J. Riveros Avances en Análisis por Técnicas de Rayos X, Vol. IX, Proceedings of the $V$ Seminario Latinoamericano de Análisis por Técnicas de Rayos $X$, edited by $\mathrm{G}$. Castellano, J. Riveros, $\mathrm{H}$. Sánchez, G. Stutz and J. Trincavelli, p. 145. Universidad Nacional de Córdoba, Córdoba, Argentina (1997).

22. E. Coleoni, X. Llovet, E. Acosta, J. García Veigas, J. Riveros and F. Salvat, Avances en Análisis por Técnicas de Rayos $X$ Vol. IX, Proceedings of the $V$ Seminario Latinoamericano de Análisis por Técnicas de Rayos $X$, edited by G. Castellano, J. Riveros, H. Sánchez, G. Stutz and J. Trincavelli, p. 163. Universidad Nacional de Córdoba, Córdoba, Argentina (1997).

23. X. Llovet, J. Riveros and F. Salvat, Mikrochim. Acta Suppl. 13, 409 (1996)

24. H. Bethe, Ann. Phy. (Leipzig) 5, 325 (1930).

25. M. Green and V. Cosslett, Proc. Phys. Soc. 78, 1206 (1961).

26. A. Vignes and G. Dez, Br. J. Appl. Phys. (J. Phys. D) 1, 1309 (1968).

27. J. Brown and L. Parobek, X-Ray Spectrom. 5, 36 (1976)

28. F. Salvat and J. Fernández-Varea, Nucl. Instrum. Methods, Sect. B 63255 (1992).

29. R. Mayol and F. Salvat, J. Phys. B 23, 2117 (1990).

30. J. Pouchou and F. Pichoir, in Proceedings of the 11th International Congress on X-Ray Optics and Microanalysis edited by J. D. Brown and R. H. Packwood, p. 249. University of Western Ontario, Ontario, Canada (1986). 